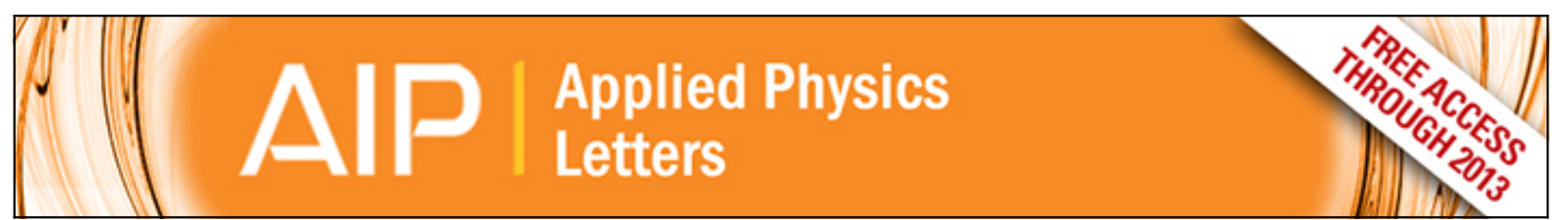

Observation of salt effects on hydration water of lysozyme in aqueous solution using terahertz time-domain spectroscopy

Katsuyoshi Aoki, Kentaro Shiraki, and Toshiaki Hattori

Citation: Applied Physics Letters 103, 173704 (2013); doi: 10.1063/1.4826699

View online: http://dx.doi.org/10.1063/1.4826699

View Table of Contents: http://scitation.aip.org/content/aip/journal/apl/103/17?ver=pdfcov

Published by the AIP Publishing

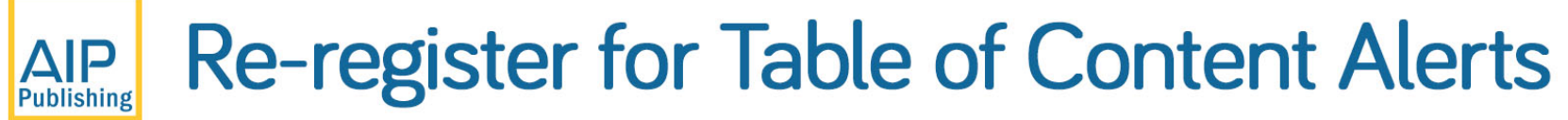

Create a profile. 


\title{
Observation of salt effects on hydration water of lysozyme in aqueous solution using terahertz time-domain spectroscopy
}

\author{
Katsuyoshi Aoki, ${ }^{1}$ Kentaro Shiraki, ${ }^{2}$ and Toshiaki Hattori ${ }^{1, a)}$ \\ ${ }^{1}$ Institute of Applied Physics, University of Tsukuba, 1-1-1 Tennodai, Tsukuba, Ibaraki 305-8573, Japan \\ ${ }^{2}$ Faculty of Pure and Applied Sciences, University of Tsukuba, 1-1-1 Tennodai, Tsukuba, Ibaraki 305-8573, \\ Japan
}

(Received 17 July 2013; accepted 11 October 2013; published online 25 October 2013)

\begin{abstract}
Terahertz time-domain spectroscopy was used to investigate the salt effect of ammonium sulfate on the dynamics of hydration water of lysozyme in aqueous solution. The absorption coefficient of lysozyme aqueous solutions containing salt was subtracted by that of the water and ammonium sulfate contained in the lysozyme solution. The results revealed that ammonium sulfate increases the absorption coefficient of the hydration water, which indicates that the dynamics of the hydration water becomes faster and/or the number of hydration water molecules decreases with increasing ammonium sulfate concentration. (C) 2013 AIP Publishing LLC. [http://dx.doi.org/10.1063/1.4826699]
\end{abstract}

Salts influence the properties of protein aqueous solutions, such as protein solubility and stability. ${ }^{1,2}$ For example, lowering the solubility by the addition of salt causes precipitation of the protein-an effect which can be used to purify the proteins. The ability to induce precipitation is particular to specific salts, the order of which is known as the Hofmeister series. ${ }^{3,4}$ According to the Hofmeister series, kosmotropic anions such as sulfate ions have greater ability to precipitate proteins. Ammonium sulfate (AS) is one particular salt that can precipitate proteins without unfolding, which has led to its industrial application as a protein precipitant. Although many studies ${ }^{2,5}$ have discussed the process of protein precipitation and stabilization, understanding the process is still challenging.

Salt effects are caused by a change in the state of the hydration water at the protein surface. ${ }^{6}$ Arakawa and Timasheff studied salt effects on hydration water of protein based on theory and experiments of preferential interaction. ${ }^{7}$ Tompa et al. reported a specific heat study of the hydration water at a protein surface using nuclear magnetic resonance (NMR) and differential scanning calorimetry (DSC). ${ }^{8}$ The dynamics of the hydration water on the picosecond timescale plays a key role in protein stability; ${ }^{9}$ however, these methods do not reveal the picosecond dynamics of hydration water but rather dynamics on the nanosecond scale and the amount of water bound to a protein.

Terahertz time-domain spectroscopy (THz-TDS) can be used to directly observe the picosecond dynamics of water molecules. This method has been applied to aqueous solutions of sugars, ${ }^{10}$ lipids, ${ }^{11,12}$ polymers, ${ }^{13}$ salts, ${ }^{14-16}$ and proteins. ${ }^{17-21}$ Havenith group has shown that the dynamics of hydration water is slower than that of bulk water using THz-TDS. $^{22,23}$ This technique can observe the hydration water that has relaxations on time scale not longer than nanosecond and that is not detected with techniques such as $\mathrm{X}$-ray scattering, neutron scattering, NMR, and calorimetry. ${ }^{10,11,22}$ In THz-TDS experiments on aqueous solutions, a

\footnotetext{
${ }^{\text {a) }}$ Author to whom correspondence should be addressed. Electronic mail: hattori@bk.tsukuba.ac.jp
}

high accuracy of measurement is required because of the strong terahertz absorption of water. High accuracy has been achieved by applying $p$-Ge laser ${ }^{20,21}$ or attenuated total reflection (ATR) methods. ${ }^{10,11}$ Here, we demonstrate measurements using a common THz-TDS setup in a transmission geometry with improved accuracy, and apply THz-TDS to investigation of the salt effect on protein hydration water.

Samples used for measurements were aqueous solutions of hen egg white lysozyme (HEWL; L6876, Sigma-Aldrich), AS (019-03435, Wako), and a lysozyme-AS mixed (LAS) solution. The HEWL and AS were used without further purification. Stock solutions of these solutes were prepared by dissolving them in pure water (Milli-Q). The concentration of the HEWL stock solution, which is determined by the absorbance change at $280 \mathrm{~nm}$ using a UV-vis spectrophotometer (ND-1000, NanoDrop Technologies, Inc., Wilmington, Del, USA), was $267 \mathrm{mg} / \mathrm{ml}$, and the concentrations of the AS stock solutions were $0,0.5,1.0$, and $1.5 \mathrm{~mol} / \mathrm{l}$. Sample solutions were prepared by mixing the HEWL and AS stock solutions at a volume ratio of $3: 1$ to obtain solutions with HEWL concentrations of $200 \mathrm{mg} / \mathrm{ml}$. No adjustment of the solution $\mathrm{pH}$ with a buffer was conducted, because the buffer ions can affect the hydration properties. The densities of the solutions were measured with a density meter (DMA 35, Anton Paar), and the amount of salt solution contained in the sample solution was calculated.

Fourier transform infrared spectroscopy in an attenuated total reflection geometry (FTIR-ATR; FT/IR-4200, Jasco, Japan) was used to confirm that no structural change of HEWL occurred in the presence of AS. The FTIR spectral profile and intensity of amide I and II peaks were found to be independent of the AS concentration (data not shown here), which indicates that the HEWL maintains the native conformation in the solutions investigated in this work. Although we attempted to confirm the secondary structure of HEWL by circular dichroism (CD), the CD measurements of the $200 \mathrm{mg} / \mathrm{ml} \mathrm{HEWL}$ solutions were difficult due to the strong optical absorption in the far-UV region. Thus, we measured the CD spectra of dilute $(1 \mathrm{mg} / \mathrm{ml})$ HEWL solutions, which showed that the secondary structure was not changed even in the presence of $0.375 \mathrm{~mol} / \mathrm{l} \mathrm{AS}$ (data not shown). 


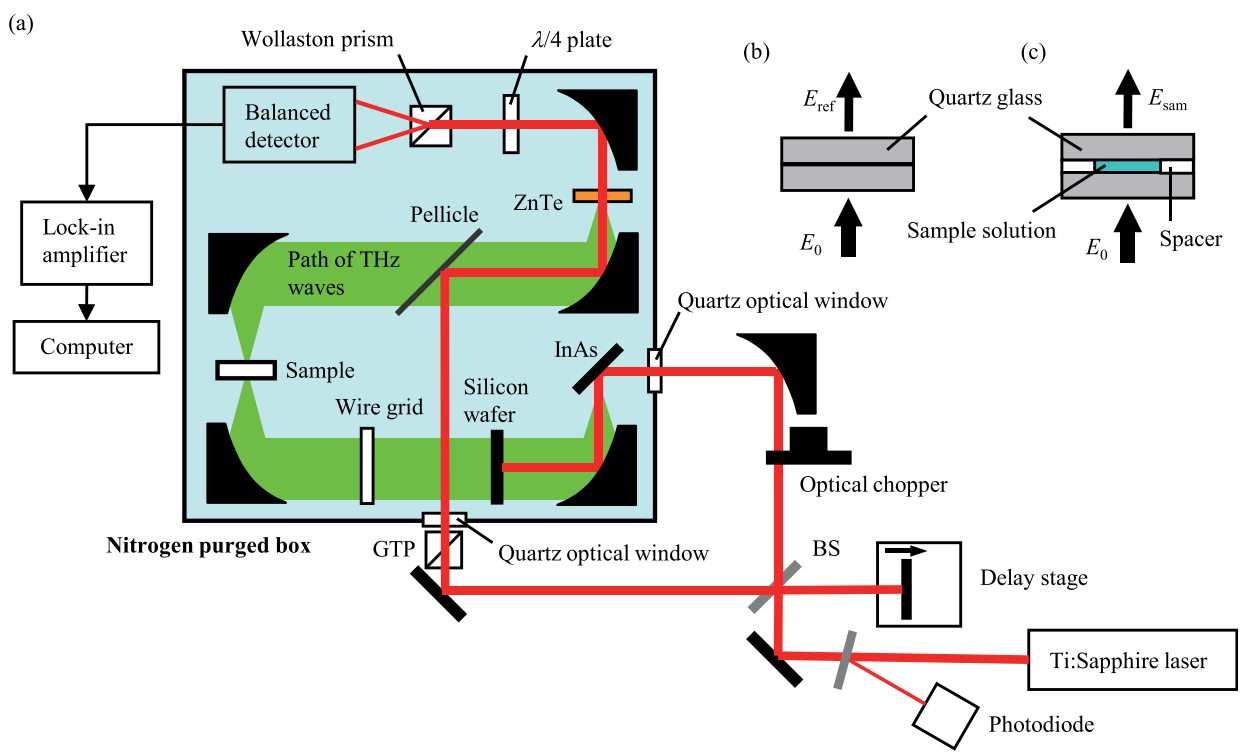

FIG. 1. Experimental setup. (a) THzTDS system, (b) glass plates for reference, (c) structure of the sample cell. BS: Beam splitter, GTP: Glan-Taylor prism.
Figure 1(a) shows the experimental setup of the THzTDS system. Optical pulses generated by a Ti:sapphire laser $(10.6 \mathrm{fs}, 470 \mathrm{~mW}, 780 \mathrm{~nm}$, Femtosource Scientific Pro, FEMTOLASERS, Austria) irradiated the (111) surface of a $p$-InAs wafer to generate terahertz pulses. ${ }^{24}$ The terahertz pulses were detected by an electro-optic method using $\mathrm{ZnTe}$ as an electro-optic crystal. The path of the terahertz waves was purged with nitrogen gas to reduce relative humidity. The temperature in the purged box was kept at $19.5 \pm 1.0^{\circ} \mathrm{C}$, and the relative humidity was under $5.0 \%$. Power fluctuations of the Ti:sapphire laser light were monitored with a photodiode and were used to correct the amplitude fluctuations of the terahertz field. Correction was performed by dividing the detected signal amplitude of the field by the square of the laser power, based on experimental results that showed the amplitude of the field signal is quadratically proportional to the laser intensity; both the generation and detection efficiency of the terahertz field should be proportional to the laser power. After this correction, the obtained peak amplitude of the terahertz pulses was almost independent of time. The structures of the sample cell for the references and sample solutions are shown in Figs. 1(b) and 1(c). The sample cell was composed of two quartz plates $(1.0 \mathrm{~mm}$ thick) and a polytetrafluoroethylene (PTFE) spacer $(0.100 \mathrm{~mm}$ thick $)$, and was sealed with Parafilm ${ }^{\circledR}$ to prevent evaporation of the sample solution. The cells for references and sample solutions were switched automatically; waveforms of the fields transmitted through these cells were measured alternatively ten times. After Fourier transformation of the waveforms, the ten amplitude ratios $t(\omega)$ and ten phase differences $\Delta \phi(\omega)$ of the fields $E_{\text {sam }}$ and $E_{\text {ref }}$ were averaged. After taking the average, the error in the absorption coefficients was below $0.6 \mathrm{~cm}^{-1}$ in the observation range of 0.3 to $1.2 \mathrm{THz}$.

Then, refractive index $n$ and absorption coefficient $\alpha$ of the solution were calculated using the following equations: ${ }^{25}$

$$
n=\frac{c}{\omega d}\left[\Delta \phi(\omega)-\arg \left\{\frac{4 \tilde{n}_{1}(\omega) \tilde{n}(\omega)}{\left(\tilde{n}_{1}(\omega)+\tilde{n}(\omega)\right)^{2}} M\right\}\right]+1,
$$

$$
\alpha=-\frac{2}{d} \log \frac{t(\omega)}{\left|\frac{4 \tilde{n}_{1}(\omega) \tilde{n}(\omega)}{\left(\tilde{n}_{1}(\omega)+\tilde{n}(\omega)\right)^{2}} M\right|},
$$

where $c$ is the speed of light in vacuum, $d$ is the thickness of the sample solution, $\tilde{n}_{1}$ is the complex refractive index of the quartz plates, $\tilde{n}$ is the complex refractive index of the sample solution, and $M$ is a correction factor for reflection effects between two interfaces of quartz plate and the sample solution represented as

$$
M=\frac{1}{1-\left(\frac{\tilde{n}-\tilde{n}_{1}}{\tilde{n}+\tilde{n}_{1}}\right)^{2} e^{2 i \omega \tilde{n} d / c}} .
$$

The complex refractive index $\tilde{n}$ is described using the real refractive index $n$ and the extinction coefficient: $\kappa=c \alpha / 2 \omega: \tilde{n}=n+i \kappa$. First, the term of the argument in Eq. (1) was set to 0 to obtain an approximate value of $n$. Using $n$ and setting $M=1$ and $\operatorname{Im}\{\tilde{n}(\omega)\}=0$ gave an approximate value of $\alpha$. More precise values of $n$ and $\alpha$ were calculated by iteration of Eqs. (1)-(3) a thousand times until sufficient convergence was achieved. To reduce uncertainty of $n$ and $\alpha$ due to the errors of HEWL concentration, the experiment shown above (from making samples to obtaining values of $n$ and $\alpha$ ) was repeated three times; the obtained values of $n$ and $\alpha$ were averaged.

Figure 2 shows the refractive indices and absorption coefficients for pure water and the HEWL $200 \mathrm{mg} / \mathrm{ml}$ aqueous solution. The refractive indices monotonically decrease with increasing frequency, while the absorption coefficients monotonically increase with the frequency. The absorption coefficient of pure water is in agreement with that previously reported. ${ }^{26}$ The refractive index and absorption coefficient of the HEWL solution were smaller than those of pure water. The observed decrease consists of contributions from (i) the decrease in the amount of water in the HEWL solution, and (ii) the decrease in the terahertz absorption of the hydration water around HEWL molecules due to their slower motion. ${ }^{27-30}$ 


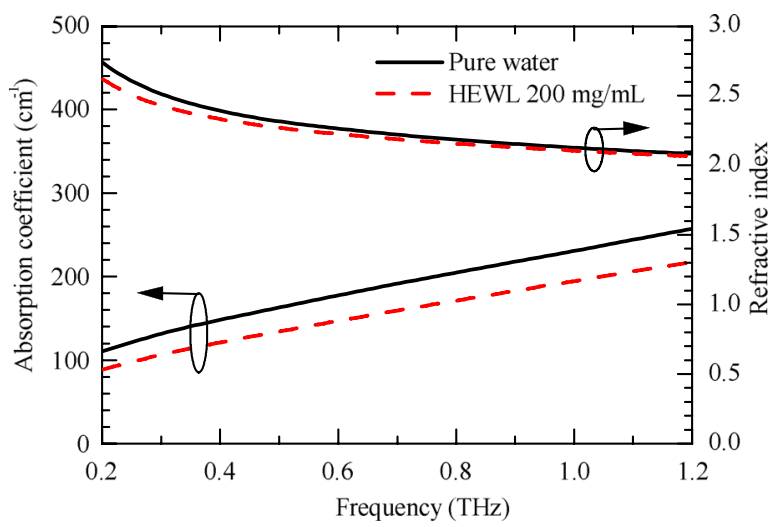

FIG. 2. Absorption coefficients and refractive indices of pure water (black solid line) and the $200 \mathrm{mg} / \mathrm{ml}$ HEWL aqueous solution (red dashed line). Errors are smaller than the line thickness.

Figure 3 shows the dependence of the absorption coefficients for the aqueous solutions on the AS concentration. The absorption coefficients of the AS and LAS aqueous solutions increase linearly with the AS concentration. Therefore, these absorption coefficients were fitted with a straight line at each frequency. The slope of these lines for the LAS aqueous solutions was twice as large as that for the AS aqueous solution at each frequency.

To evaluate the change in the dynamics of the HEWL hydration water, the absorption coefficient of AS aqueous solution contained in the LAS solution (second term on the right side of Eq. (4)) was subtracted from the total absorption coefficient of the LAS solution $\alpha_{\text {LAS }}$ (first term on the right side of Eq. (4)) at each frequency

$$
\Delta \alpha=\alpha_{\mathrm{LAS}}-\frac{\rho_{\mathrm{LAS}}-c_{\mathrm{HEWL}}}{\rho_{\mathrm{AS}}} \alpha_{\mathrm{AS}},
$$

where $\rho_{\text {AS }}$ is the density of the AS solution, $\rho_{\text {LAS }}$ is the density of the LAS solution, and $c_{\text {HEWL }}$ is the HEWL concentration in the LAS solution. The linear-fitting parameters obtained in Fig. 3 were used for this calculation. This analysis, which uses the densities of the AS and LAS solutions, can eliminate effects of difference of mass fractions of water between these solutions. The $\Delta \alpha$ spectra obtained are shown

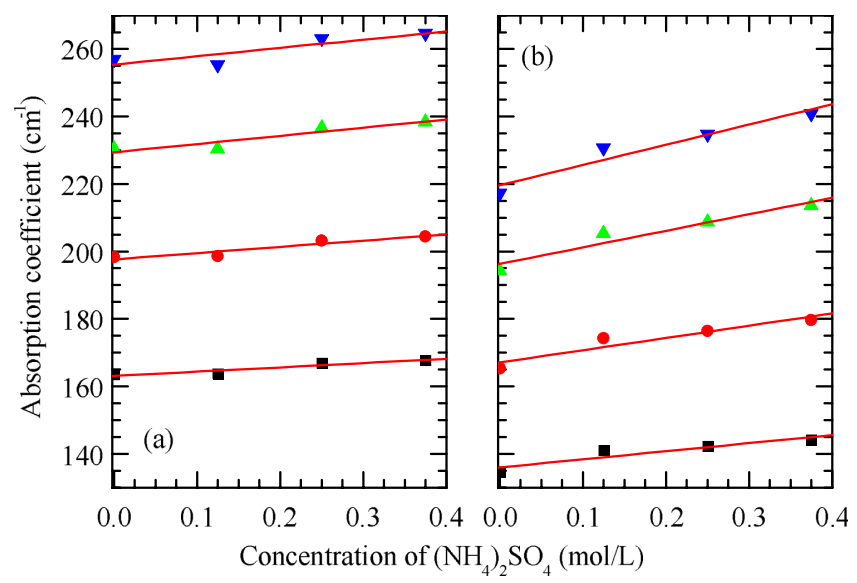

FIG. 3. Dependence of the absorption coefficients of (a) AS aqueous solutions and (b) LAS aqueous solutions on the AS concentration at $0.50 \mathrm{THz}$ (black square), $0.75 \mathrm{THz}$ (red circle), $1.0 \mathrm{THz}$ (green triangle), and $1.2 \mathrm{THz}$ (blue inverted triangle). Error bars are smaller than the symbols.

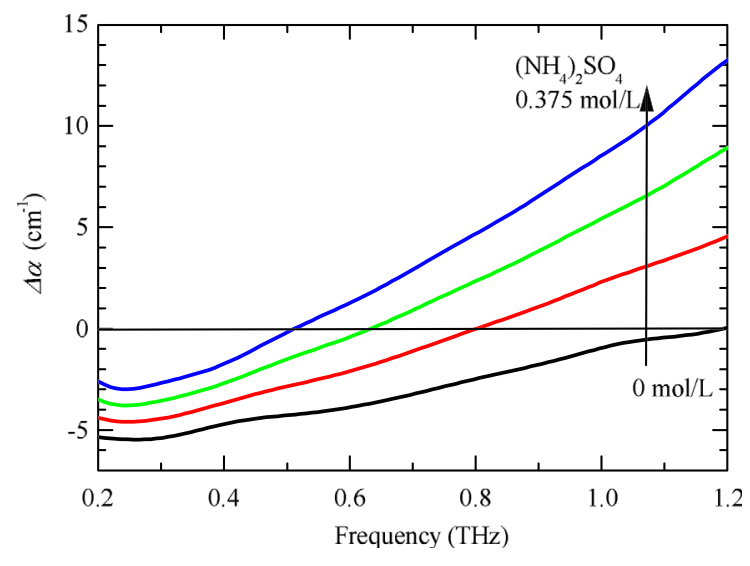

FIG. 4. Absorption coefficients for LAS solutions by subtraction of that for the AS aqueous solution using Eq. (4). The concentration of HEWL was $200 \mathrm{mg} / \mathrm{ml}$. These curves were obtained using the linear-fitting parameters obtained in Fig. 3.

in Fig. 4. For solutions without $\mathrm{AS}, \Delta \alpha$ is negative, which is attributed to the slowing down of the hydration water dynamics at the HEWL surface. ${ }^{27}$ Addition of AS increases $\Delta \alpha$, due to an increase in the terahertz absorption of the HEWL hydration water because the HEWL maintains the native conformation. This results indicates that the dynamics of the HEWL hydration water becomes faster and/or that the amount of HEWL hydration water decreases.

The number of hydration water molecules $N$, namely, the number of water molecules in the dynamical hydration shell of a HEWL molecule, was calculated according to the method reported in Ref. 27, where it is assumed that the hydration water has no terahertz absorption and that HEWL has no absorption at the minimum frequency of $\Delta \alpha{ }^{27} \mathrm{We}$ note that the calculation of $N$ is based on a simple model composed by bulk water, water in the hydration shells and the HEWL molecules. In the actual solutions, there may not be strict separation between bulk water and hydration water. Obtained $N$ is, therefore, an effective value describing the decrease in the mobility of water molecules surrounding the HEWL molecule. The relation between the AS concentration $c_{\text {AS }}$ and $N: N=(182 \pm 30)-(201 \pm 130) c_{\text {AS }}$ was obtained, where the unit of $c_{\mathrm{AS}}$ is $\mathrm{mol} / \mathrm{l}$.

Vinh et al. $^{27}$ reported that the number of HEWL hydration waters was $153 \pm 15$ using dielectric spectroscopy from 0.065 to $0.7 \mathrm{THz}$. However, sodium phosphate buffer was added to the HEWL aqueous solution in their experiment. In the present work, buffer was not intentionally added, but the HEWL that was used contains buffer salts such as sodium acetate and sodium chloride (less than 10\%). The difference and ambiguity in the amount of buffer salts can affect the obtained $N$, although it should not significantly affect the AS concentration dependence of $\Delta \alpha$. The present finding that $\Delta \alpha$ increases ( $N$ decreases) with AS concentration is consistent with Collins' model of protein hydration, ${ }^{31}$ which shows that AS, known as a structure-making salt, ${ }^{1}$ makes hydration of the protein weaker.

In summary, THz-TDS was applied to investigate and observe salt effects on the dynamics of HEWL hydration water. Accuracy in the measurement of the absorption coefficient and refractive index was improved by monitoring the 
laser power and by taking averages of $t(\omega)$ and $\Delta \phi(\omega)$ obtained from ten delay-time scans. The dynamics of the HEWL hydration water was confirmed to be slower than that of bulk water, which is in good agreement with previous studies. ${ }^{27}$ We have revealed that the addition of AS linearly increases the absorption coefficient of the HEWL hydration water. The decrease rate in the number of water molecules in the dynamical hydration shell of a HEWL molecule was determined to be $201 /(\mathrm{mol} / \mathrm{l})$ using our analytical method. These results indicate that the dynamics of HEWL hydration water becomes faster and/or the number of HEWL hydration water molecules decreases by the addition of AS. THz-TDS and the present analytical methods are useful for the study of not only salt effects on the protein hydration water but also the effects of other types of additives such as denaturants, structural stabilizers, and aggregation suppressors. This is a key technology to investigate phenomena in solutions, such as the Hofmeister effect, with respect to the hydration water.

The authors would like to thank Professor Hideaki Kano (University of Tsukuba) for useful discussions and Ms. Chie Tanaka (University of Tsukuba) for experimental support.

${ }^{1}$ Y. Zhang and P. S. Cremer, Curr. Opin. Chem. Biol. 10, 658 (2006).

${ }^{2}$ K. D. Collins and M. W. Washabaugh, Q. Rev. Biophys. 18, 323 (1985).

${ }^{3}$ F. Hofmeister, Arch. Exp. Pathol. Pharmakol. 24, 247 (1888).

${ }^{4}$ W. Kunz, J. Henle, and B. W. Ninham, Curr. Opin. Colloid Interface Sci. 9, 19 (2004).

${ }^{5}$ R. L. Baldwin, Biophys. J. 71, 2056 (1996).

${ }^{6}$ E. Sedlák, L. Stagg, and P. Wittung-Stafshede, Arch. Biochem. Biophys. 479, 69 (2008).

${ }^{7}$ T. Arakawa and S. N. Timasheff, Biochemistry 23, 5912 (1984).

${ }^{8}$ K. Tompa, P. Bánki, M. Bokor, P. Kamasa, G. Lasanda, and P. Tompa, Biophys. J. 96, 2789 (2009).
${ }^{9}$ S. K. Pal, J. Peon, and A. H. Zewail, Proc. Natl. Acad. Sci. U.S.A. 99, 1763 (2002).

${ }^{10}$ T. Arikawa, M. Nagai, and K. Tanaka, Chem. Phys. Lett. 457, 12 (2008).

${ }^{11}$ M. Hishida and K. Tanaka, Phys. Rev. Lett. 106, 158102 (2011).

${ }^{12}$ D.-H. Choi, H. Son, S. Jung, J. Park, W.-Y. Park, O. S. Kwon, and G.-S. Park, J. Chem. Phys. 137, 175101 (2012).

${ }^{13}$ H. Naito, Y. Ogawa, H. Hoshina, S. Sultana, and N. Kondo, Appl. Phys. Lett. 100, 191102 (2012).

${ }^{14}$ J. Xu, K. W. Plaxco, S. J. Allen, J. E. Bjarnason, and E. R. Brown, Appl. Phys. Lett. 90, 031908 (2007).

${ }^{15}$ K. J. Tielrooij, N. Garcia-Araez, M. Bonn, and H. J. Bakker, Science 328, 1006 (2010).

${ }^{16}$ J. Xu, K. W. Plaxco, and S. J. Allen, J. Chem. Phys. 124, 036101 (2006).

${ }^{17}$ J. Xu, K. W. Plaxco, and S. J. Allen, Protein Sci. 15, 1175 (2006).

${ }^{18}$ J. Xu, K. W. Plaxco, and S. J. Allen, J. Phys. Chem. B 110, 24255 (2006).

${ }^{19}$ M. Heyden and M. Havenith, Methods 52, 74 (2010).

${ }^{20}$ S. Ebbinghaus, S. J. Kim, M. Heyden, X. Yu, M. Gruebele, D. M. Leitner, and M. Havenith, J. Am. Chem. Soc. 130, 2374 (2008).

${ }^{21}$ S. Ebbinghaus, S. J. Kim, M. Heyden, X. Yu, U. Heugen, M. Gruebele, D. M. Leitner, and M. Havenith, Proc. Natl. Acad. Sci. U.S.A. 104, 20749 (2007).

${ }^{22}$ U. Heugen, G. Schwaab, E. Bründermann, M. Heyden, X. Yu, D. M. Leitner, and M. Havenith, Proc. Natl. Acad. Sci. U.S.A. 103, 12301 (2006).

${ }^{23}$ M. Heyden, E. Bründermann, U. Heugen, G. Niehues, D. M. Leitner, and M. Havenith, J. Am. Chem. Soc. 130, 5773 (2008).

${ }^{24}$ R. Adomavičius, A. Urbanowicz, G. Molis, A. Krotkus, and E. Šatkovskis, Appl. Phys. Lett. 85, 2463 (2004).

${ }^{25}$ C. Zhang, E. Tarhan, A. K. Ramdas, A. M. Weiner, and S. M. Durbin, J. Phys. Chem. B 108, 10077 (2004).

${ }^{26}$ M. N. Afsar and J. B. Hasted, J. Opt. Soc. Am. 67, 902 (1977).

${ }^{27}$ N. Q. Vinh, S. J. Allen, and K. W. Plaxco, J. Am. Chem. Soc. 133, 8942 (2011).

${ }^{28}$ F. Sterpone, G. Stirnemann, and D. Laage, J. Am. Chem. Soc. 134, 4116 (2012).

${ }^{29}$ M. Marchi, F. Sterpone, and M. Ceccarelli, J. Am. Chem. Soc. 124, 6787 (2002).

${ }^{30}$ S. Perticaroli, L. Comez, M. Paolantoni, P. Sassi, L. Lupi, D. Fioretto, A. Paciaroni, and A. Morresi, J. Phys. Chem. B 114, 8262 (2010).

${ }^{31}$ K. D. Collins, Methods 34, 300 (2004). 